\title{
REVUE DES INTRODUCTIONS DE POISSONS ET DE CRUSTACÉS DÉCAPODES D'EAU DOUCE EN POLYNÉSIE FRANÇAISE.
}

\author{
P. KEITH \\ Muséum National d'Histoire Naturelle, laboratoire d'ichtyologie, 43 rue Cuvier, \\ 75231 PARIS Cedex 05, France.
}

Reçu le 23 janvier 2002

Received 23 January, 2002

Accepté le 22 février 2002

Accepted 22 February, 2002

\section{RÉSUMÉ}

La faune dulçaquicole indigène ou introduite de Polynésie française a été méconnue jusqu'à la fin du $20^{\text {ème }}$ siècle, qui a vu les premiers inventaires exhaustifs du Muséum National d'Histoire Naturelle. Les introductions d'espèces ont commencé en 1920 avec le guppy pour atteindre finalement 11 espèces, qui se sont plus ou moins acclimatées. Seul l'archipel des Marquises est exempt d'espèces introduites. Les trois autres pourvus de rivières, les archipels de la Société, des Gambier et des Australes possèdent 1 à 3 espèces acclimatées et 1 à 3 espèces présentes dans des fermes aquacoles et que l'on pourrait retrouver dans les rivières durant les prochaines années. d'espèces.

Mots-clés : poissons, crustacés, eau douce, Polynésie française, introduction

\author{
INTRODUCTION OF FRESHWATER FISHES \\ AND DECAPOD CRUSTACEANS IN \\ FRENCH POLYNESIA, A REVIEW.
}

\begin{abstract}
The autochtonous or introduced freshwater fauna of French Polynesia was poorly known up to the end of the $20^{\text {th }}$ century. The introduction of freshwater fishes and crustaceans began in 1920 with the introduction of the guppy and finally 11 species were introduced with more or less results. The Marquesas Archipelago is the only one without introduced species. The three others with perennial streams, the Society, Gambier and Australes Archipelagos, have one to three acclimatised species and one to three species present in aquaculture farm and which could be found soon in freshwater.
\end{abstract}

Key-words : fishes, crustaceans, freshwater, French Polynesia, species introduction. 


\section{INTRODUCTION}

La Polynésie française couvre un vaste domaine océanique situé à la limite est de la province Indo-Pacifique. Elle est formée de 118 îles, comprenant des îles hautes volcaniques et des îles basses coralliennes ou atolls. Ces différentes îles représentent une surface de $3629 \mathrm{~km}^{2}$ de terres émergées, disséminées sur 2,5 millions de $\mathrm{km}^{2}$ d'océan. Elles sont réparties en cinq archipels, dispersés le long d'un axe général sud-est, nordouest. Ce sont :

- l'archipel des Australes $\left(141 \mathrm{~km}^{2}\right)$ : six îles hautes et un atoll ;

- l'archipel des Gambier (23 km²) : neuf îles hautes ;

- l'archipel des Marquises $\left(997 \mathrm{~km}^{2}\right)$ : onze îles hautes et un atoll ;

- l'archipel de la Société (1618 $\left.\mathrm{km}^{2}\right)$ : neuf îles hautes et cinq atolls ;

- l'archipel des Tuamotu $\left(850 \mathrm{~km}^{2}\right): 76$ atolls.

Seuls les 4 premiers archipels possèdent des rivières pérennes susceptibles d'abriter une faune dulçaquicole. Cette faune d'eau douce, qu'il s'agisse d'espèces indigènes ou non, était jusqu'à une période encore très récente, largement inconnue. En effet, bien que les inventaires de poissons réalisés sur ce Territoire d'Outre-Mer eurent débuté à la fin du siècle dernier, ils furent alors essentiellement consacrés aux poissons marins. La faune d'eau douce était très peu étudiée (KEITH et al., 2002). On trouve néanmoins quelques informations dans des articles consacrés essentiellement à la faune marine : POLL (1942) et RANDALL (1973a, b) pour la Société, PLESSIS et MAUGÉ (1978) pour les Marquises, FOURMANOIR et al. (1974) pour les Gambier et PLESSIS (1987), PLESSIS (1980) et RANDALL et al. (1990) pour les Australes.

Peu d'articles ont été exclusivement consacrés à la faune d'eau douce jusqu'aux années 1985-1990 ; on note cependant ceux de FOWLER (1932), de SEURAT (1934), de EGE (1939) et surtout ceux de MARQUET et GALZIN (1992) et de MARQUET (1993). Aucune publication ne fut consacrée aux introductions d'espèces. Néanmoins, dans le premier quart du $20^{\text {ème }}$ siècle, la Société des Etudes Océaniennes (S.E.O.) relate parfois dans son bulletin les tentatives qui ont été réalisées.

Les publications consacrées spécifiquement aux crustacés d'eau douce de Polynésie française sont également rares dans la littérature. SEURAT (1934) signalait 5 espèces à Tahiti et 3 espèces à Mangareva (Gambier), ADAMSON (1935) en signalait 6 aux Marquises et EDMONSON (1935) a décrit une nouvelle caridine de l'île de Rapa. Des travaux généraux de révision ou de biogéographie feront référence aux espèces présentes en Polynésie (BOUVIER, 1925 ; EDMONSON, 1951 ; HOLTHUIS, 1950, 1952, 1980 ; HOBBS et HART, 1982) mais il faut attendre 1991 (MARQUET, 1991) pour la première synthèse des connaissances disponibles.

Enfin, dans la période 1997-2001, des inventaires exhaustifs (MNHN/EPHE) ont été réalisés par KEITH et VIGNEUX (1997), KEITH et al. (2000) et KEITH et al. (2002) sur les îles de l'Archipel de la Société, des Marquises et des Australes. Ces inventaires furent à l'origine de la découverte de plusieurs espèces nouvelles pour la science (KEITH et VIGNEUX, 2002), mais aussi de la découverte d'espèces anciennement introduites et oubliées.

Cet article se propose de faire le bilan des introductions d'espèces dulçaquicoles en Polynésie française. 


\section{HISTOIRE DES INTRODUCTIONS EN POLYNÉSIE FRANÇAISE}

En 1926, dans un article du Bulletin de la Société d'Etude Océanienne (S.E.O.), Mr Rougier rédigeait une courte note sur les 5 espèces de poissons d'eau douce connues à l'époque en Polynésie française. II concluait ainsi et posait les bases de ce qui allait conduire aux premières introductions de poissons :

"...Voilà donc toutes les richesses aquatiques de nos cours d'eau, pourtant si nombreux, et la conclusion c'est que nos ressources en poissons d'eau douce sont presque nulles. De cette conclusion naissent les réflexions suivantes :

Nos cours d'eau, étangs et estuaires, valent-ils la peine d'essayer la culture ou l'introduction de certains poissons? Nous savons que nos cours d'eau sont nombreux, que leur eau est vive et intarissable dans les hauteurs et que, chose appréciable, beaucoup sont inaccessibles, ce qui en fait forcément des pêches gardées. Par ailleurs, nous avons des estuaires, des étangs ou marais très nombreux, jamais très grands mais suffisants quand même, pour bien des tonnes de poissons. Leurs fonds sont vaseux, quelquefois profonds, mais toujours herbeux, fournissant un habitat idéal pour certaines espèces.

La température de nos eaux en montagne va de $10^{\circ}$ à $20^{\circ}$ à partir de $1000 \mathrm{~m}$ jusqu'à $1500 \mathrm{~m}$ d'altitude pour atteindre 21 à $28^{\circ}$ en s'approchant de la mer. Nos estuaires et nos étangs ne descendent jamais au-dessous de $25^{\circ}$. Tout ceci constaté, la question se pose: existe-t-il des poissons pouvant vivre et se multiplier dans les conditions cidessus ?... » (ROUGIER, 1926a).

\section{Carpe, Black-bass, Poisson-chat et Gambusie}

Dès 1925, le Bureau de Pisciculture des Etats-Unis à San Francisco avait été consulté par la S.E.O.. M.J.F. Hunter, chef de ce bureau, avait alors conseillé d'introduire la truite arc-en-ciel (Oncorhynchus mykiss (Walbaum, 1792)) dans les cours d'eau de montagne de Polynésie française (KEITH et al., 2002).

Le Dr. Barton Warren Evermann, une des plus grandes autorités en pisciculture de l'époque et chef de l'Aquarium de Golden Park (San Francisco), avait conseillé, quant à lui, des ombles (Salvelinus sp.) pour les zones montagneuses. Tous deux étaient d'avis que tanches (Tinca tinca (Linné, 1758)), carpes (Cyprinus carpio Linné, 1758) et poissonschats (Ictalurus sp.) devraient s'acclimater dans les étangs et les estuaires. Ces deux institutions avaient promis d'envoyer gracieusement des œufs de truites au moment du frai.

C'est la même année que le Gouverneur Rivet a bien voulu mettre à la disposition de la S.E.O. la moitié des sources dites « de la Reine " et une petite installation de pisciculture fut bientôt prête à recevoir les œufs promis (KEITH et al., 2002).

En novembre 1925, le Dr Evermann et son assistant M. A. Seale firent parvenir à Tahiti par le bateau "Makura » un premier envoi de carpes et de poissons-chats de l'Aquarium. Trente-six poissons de chacune de ces espèces furent expédiés. Mr Dalgliesh, premier officier à bord, se chargea lui-même de renouveler quatre fois par jour l'eau des grands barils qui servaient de réservoirs. Malgré cela, deux carpes seulement arrivèrent vivantes et un seul poisson-chat Maraa (ROUGIER, 1926a et 1926b). En revanche, plus de 200 gambusies (Gambusia affinis Baird et Girard, 1853) arrivèrent en bon état. La Gambusie a été introduite sur tous les continents (sauf l'Antarctique) pour contrôler les moustiques susceptibles de transmettre la malaria.

Devant cet échec, de nouvelles dispositions furent prises. Sur le " R.M.S. Makura ", dont un nouveau départ était prévu en janvier 1926, furent installées à grands frais à San Francisco, 4 grandes tinques en fer. Chacune était reliée par un tube en caoutchouc à un 
immense réservoir d'oxygène, de telle sorte que le renouvellement de l'eau dans les tinques devenait inutile. Le tout était offert gracieusement par Mr Back, Directeur à San Francisco de l'Union Steam Ship Compagny (KEITH et al., 2002).

Cette fois-ci, de nombreux spécimens arrivèrent vivants ce qui permit à la S.E.O. de procéder à une distribution des poissons aux divers membres de la Société qui avaient des bassins. Ainsi, furent répartis : 50 carpes, 30 black-bass et 12 poissons chats. Ce fut la première introduction du black-bass (Micropterus salmoides Lacépède, 1802) sur le territoire (ROUGIER, 1926a, 1926b, 1928), alors qu'il avait été importé dès 1897 à Hawaï.

Un premier bilan de ces introductions fut donné par Rougier en 1926 où il indiquait :

"Nos acquisitions : Grenouilles - Le "Makura" du mois de mars à apporté 34 grenouilles qui ont été immédiatement lâchées, bien vivantes, en contrebas des sources de la Reine, chez M. le Gouverneur ; or, on n'en a pas revu une seule. Poissons antimoustiques - Nous en avons de deux sortes : les guppy ou arc-en-ciel ou encore " million fish " et les gambusies. Les premiers introduits par M. Guild de Maraa et les autres envoyés par l'aquarium de San Francisco (Steinhart Aquarium) à notre Société... II semble que le guppy se reproduise ici plus vite que la Gambusie... On peut se procurer de ces poissons chez les membres suivants de notre Société : MM. le Président et Vice-Président, Emile Lévy et Barrier. Carpes et bass - Ils continuent à faire espérer que ce sera un succès, ni mort, ni maladie et ils grossissent très vite. Poissons-chats - La douzaine introduite semble moins se plaire sous notre climat que les carpes et il faut s'en réjouir car celles-ci valent mieux que ceux-là. Que va nous rapporter le Makura: tanches, carpes ou truites?».

En septembre 1926, 63 poissons moustiques furent libérés dans le lac Vahiria.

En 1928, Rougier indiquait : « il ne faut pas mettre de Gambusia, là où l'on veut conserver des chevrettes et des poissons, car il dévore tout ce qui est minuscule, larves et œufs de toutes sortes, il s'attaque même à ce qui a vie. C'est ainsi qu'il dévore ses propres petits aussitôt après leur naissance... En 1927, œufs et alevins de carpes furent dévorés, dans mon bassin, par les Gambusia qui y étaient millions ; comme je viens de détruire ceux-ci, j'espère un succès pour 1928... Poissons-chats, black-bass, grenouilles, il n'en reste plus. Je blâme les anguilles. ”

Finalement, les carpes, poissons-chats, black-bass ne s'acclimatèrent pas. Quant aux gambusies, il semble qu'elles aient été supplantées par les guppys. Les expériences des membres de la S.E.O. semblent s'être arrêtées vers la fin des années 1920 (KEITH et al., 2002).

\section{Le Guppy}

Le guppy (Poecilia reticulata, Peters, 1859) semble avoir été introduit en Polynésie française dans les années 1920 par Mr Guild de Maraa (ROUGIER, 1926c). C'est un poisson tropical capable de survivre (à des températures inférieures à $15^{\circ} \mathrm{C}$ ) dans des eaux saumâtres, ainsi que dans des eaux peu oxygénées. Ces capacités adaptatives, alliées à sa grande fécondité et à sa maturité sexuelle précoce, lui ont permis d'étendre rapidement son aire de répartition. Utilisé pour contrôler les larves de moustiques, il est aussi très prisé en aquariophilie en raison des couleurs arborées par les mâles. Ceci a contribué à son extension géographique à travers le globe. L'espèce est assez répandue en Polynésie française (Société, Australes) (KEITH et al., 2002). 


\section{Le Molly}

Le molly (Poecilia sphenops Val. in Cuvier et Val., 1846) a été capturé sur certaines rivières des îles de Tahiti, de Huahine, de Raiatea et de Taaha (KEITH et al., 2002). La date d'introduction et l'origine du stock sont inconnues mais il est probable que son acclimatation en rivière a commencé au début des années 1970. En effet, l'élevage du Molly fut mis en place en Polynésie à cette époque afin de fournir des vifs pour la pêche aux thonidés (UWATE et al., 1984). L'utilisation des molly comme appâts a été encouragée à plusieurs reprises (SPC, 1975, 1978). A Tahiti c'est le CNEXO qui l'a initiée (CROSSLAND et GRANDPERRIN, 1979 ; KENT, 1980). Ils furent élevés en cages flottantes en mer. Finalement, l'espèce s'est révélée être un piètre appât (KEITH et al., 2002). En revanche, elle est fréquemment utilisée pour contrôler les populations de moustiques sur les îles du Pacifique.

\section{La truite arc en ciel}

Le « Makura » du 26 juin 1927 débarquait à Tahiti 10000 œufs de truite arc-en-ciel (Oncorhynchus mykiss (Walbaum, 1792)), don généreux de Mr Marx Greene, Chef de la "Fish \& Game Commission of California » (FOWLER, 1925 ; ROUGIER, 1926a, 1926b, 1928).

Les œufs avaient déjà plus d'un mois d'incubation, ils étaient de la grosseur d'un petit pois et on distinguait les yeux des alevins. Une période de 20 jours environ était encore nécessaire avant l'éclosion. Des incubateurs avaient été préparés aux "sources de la Reine ", selon les connaissances de l'époque : obscurité, arrivée d'eau graduée de bas en haut à travers des cadres-treillis pour les œufs plongés à la profondeur optimale, etc., rien ne manquait... excepté le degré de fraîcheur voulu. En effet, cette source, réputée fraîche, avait une température régulière de $24^{\circ} \mathrm{C}$. Le problème de la S.E.O. était de réduire de $12^{\circ} \mathrm{C}$ l'eau qui coulait à $24^{\circ} \mathrm{C}$ afin de la rendre compatible avec les exigences biologiques de l'espèce (KEITH et al., 2002).

Les œufs arrivèrent sur 7 cadres disposés par étage et entourés de glace dans une caisse spéciale à ces transports. La glace en avait été renouvelée tous les jours à bord du "Makura ». La S.E.O. raconte ainsi la suite des événements : " Une fois en possession des œufs, notre première visite fut pour la glacière. Son directeur Mr Ferman mit gracieusement et gratuitement à notre disposition toute la glace dont nous pourrions avoir besoin, ce jour là et les jours suivants. Arrivés à la pisciculture, le thermomètre déposé sur les cadres indiqua $4^{\circ}$. Notre glace plongée en abondance dans les incubateurs, eut vite fait de ramener leur température de $24^{\circ}$ à $5^{\circ}$, mais à condition d'empêcher l'eau d'y couler. Les œufs furent donc transférés avec précaution dans un milieu moins froid de $1^{\circ}$. Notre plan était d'arriver progressivement et insensiblement à $12^{\circ}$ et de maintenir cette même température jusqu'à l'éclosion. Les œufs furent trouvés en parfait état, tous d'un beau rose.

A 14h, nous avions déjà fait deux voyages à la glacière ; la glace fondait comme neige au soleil car l'eau arrivait directement sur cette glace à raison de 1 litre 1/2 par minute. A 16h, il devint évident qu'il fallait un minimum de $20 \mathrm{~kg}$ de glace toutes les deux heures. C'est-à-dire qu'il aurait fallu veiller toute la nuit et tenir la glacière ouverte ! C'était l'impossible. Aussi après un dernier chargement de glace, il fut décidé d'abandonner les $œ u f s$ à leur sort. A 17h, le cœur navré, nous quittâmes la pisciculture. Le lendemain, au point du jour, les œufs de roses étaient devenus blancs. C'était la mort. Après trois jours, ils s'agglutinaient, il fallut les jeter.

Mr Ferman, informé, eut la bonté de nous consoler en nous promettant une salle noire dans sa glacière pour 1928, avec tuyaux traversant nos incubateurs pour donner à 
l'eau la température voulue. Mr. Ferman ne fut pas d'ailleurs le seul à s'intéresser à cet essai. Mr Sigogne, au nom du syndicat d'initiative, nous avait fait parvenir 400 F. M. Bach, directeur général de l'Union Steam Ship and Compagny avait fait transporter gratis ces œufs par le «Makura».

MM. Strange et Coryell, nos actifs membres correspondants de San-Francisco, avaient fait toutes les démarches nécessaires pour cet envoi. II paraît qu'autrefois des truites furent introduites avec succès dans les hauts de la Fautaua, mais quand et comment reste un secret.

Ce récit a donc été fait pour que la relation d'une pareille expérience reste cette fois consignée dans nos annales. Espérons que cet échec ne découragera personne, ni nos amis de San Francisco, ni nous-mêmes et disons à 1928 »(Rougier, 1926a, 1926b).

L'expérience fut donc renouvelée en 1928 où les membres correspondants de la S.E.O. de San Francisco, MM. Strange et Coryell, avaient obtenu 25000 œufs de truites arc-en-ciel. Ces œufs arrivèrent le 23 juin 1928 par le « Tahiti » et ils furent immédiatement déposés à la glacière où Mr Ferman avait organisé une petite pisciculture dans une des chambres noires. Les œufs furent divisés en deux parties. Les douze mille premiers œufs furent déposés dans deux bacs sur des claies spéciales (ROUGIER, 1926a, 1926b, 1928). Une eau claire cascadait d'un bac dans l'autre, à raison de 1 à 2 litres par minute. La température de l'eau était constante à $7^{\circ} \mathrm{C}$. Malgré tous les soins dont ils furent l'objet, les œufs survécurent moins d'une semaine sans que la cause de leur mort ne fut expliquée (KEITH et al., 2002).

Le deuxième lot fut transporté le mercredi 27 juin dans les hauts de Fautaua par 3 prisonniers sous la direction de M. Guého. Le transport se fit dans une caisse aménagée de telle sorte que les claies reçues de San Francisco étaient entourées de glace. Ils furent déposés dans trois parcs faits dans le lit de la Fautaua, dans l'obscurité d'une falaise et d'arbrisseaux, à $500 \mathrm{~m}$ d'altitude. Tous les œufs furent répandus sur des cailloux et graviers très propres où l'eau arrivait à profusion à travers les pierres des parcs. La température de l'eau n'excédait jamais $20^{\circ} \mathrm{C}$ à midi (ROUGIER, 1926a, 1926b, 1928).

Les clôtures de ces parcs sortaient de l'eau de 15 à $18 \mathrm{~cm}$ afin d'empêcher chevrettes et anguilles de venir troubler l'incubation. Ceci permettait aussi de couvrir ces parcs de branchages destinés à tenir les œufs dans une plus grande obscurité. Huit jours après, jour pour jour, la plupart des alevins étaient nés. 15 jours plus tard, il restait à peine quelques sujets traînant péniblement leur jaune vésicule ombilicale, puis bientôt plus rien (KEITH et al., 2002).

Les expériences importantes concernant la truite arc-en-ciel se sont pratiquement arrêtées à ce stade et l'espèce ne s'est jamais acclimatée.

\section{Le tilapia du Mozambique}

En raison de leur capacité à agir comme agent de contrôle biologique et de leur facilité d'élevage, plusieurs espèces de tilapia ont été introduites à partir de 1950 dans de nombreuses îles du Pacifique. Bien que restreints au départ à des bassins ou à des étangs, les tilapias du Mozambique (Peters, 1852) ont rapidement colonisé les eaux libres après y avoir été relâchés accidentellement ou volontairement. Le tilapia du Mozambique, Oreochromis mossambicus s'est ainsi acclimaté dans des lacs, des rivières, des lagons, des mangroves et des marais, tant sur des atolls coralliens, que sur des îles volcaniques.

Toutes les introductions de tilapia du Mozambique faites dans le Pacifique sud proviennent (a priori) d'une seule source : un estuaire d'une rivière à l'ouest de Java où, en 1939, Jan Mudjair, un technicien des pêches néerlandais des Antilles découvrit 
5 tilapias. Plus tard, après que ces poissons eussent été utilisés en aquaculture, les forces armées japonaises en introduisirent à Singapour et en Thaïlande en 1943, puis de là, en 1950 aux Philippines (KEITH et al., 2002).

La combinaison de facilités d'accès (le transport aérien entre Manille et Singapour et les îles du Pacifique sud ne prend que quelques heures) et d'une publicité importante (vis à vis du tilapia) effectuée d'abord par la FAO puis relayée par la Commission du Pacifique Sud (CPS), ont été les facteurs primordiaux du succès de l'implantation de l'espèce dans cette région.

L'espèce fut introduite à plusieurs reprises dans les années 1950 à Tahiti pour fournir une source supplémentaire de protéines. Elle fut notamment introduite en 1957 dans deux bassins par un éleveur privé à Taravao Tahiti (DEVAMBEZ, 1964 ; MACIOLEK, 1984). Elle fut aussi élevée par les services de pêche locaux pour servir d'appât en mer, mais elle fut reconnue comme étant peu efficace (VAN PEL et DEVAMBEZ, 1957). L'espèce s'est répandue en Polynésie et a été retrouvée dans les archipels de la Société, des Australes et des Gambier (KEITH et al., 2002).

\section{Le Barramundi}

Le Barramundi (Lates calcarifer (Bloch, 1790)) a été introduit et acclimaté pour l'aquaculture dans les eaux littorales de Tahiti (FUCHS, 1987 ; PRESTON, 1990 ; ELDREDGE, 1994). L'Ifremer à Taravao abrite, dans des cages en mer, un élevage de cette espèce ; celle-ci ne semble pas pour l'instant avoir colonisé les eaux douces (KEITH et al., 2002).

\section{Le Chanos}

L'élevage du Chanos, Chanos chanos (Forsskål, 1775), fut lancé en 1971 au village de Avatoru. En février 1976, il fut expérimenté dans l'atoll de Rangiroa à Pavete par l'intermédiaire du Service de la Pêche de Polynésie en association avec la banque Socredo, sur 1,5 hectares de zones saumâtres (BROWN, 1976 ; TOKORAGI, 1978). L'archipel des Tuamotu avait été considéré comme idéal pour l'élevage de cette espèce en raison de ses nombreux lagons. L'élevage en est toujours resté pratiquement au stade expérimental (KEITH et al., 2002).

\section{La chevrette}

La chevrette Macrobrachium rosenbergii (Günther, 1866) fut introduite d'Hawaï vers Tahiti au début des années 1970 pour une étude préliminaire (VILLALUZ, 1972), en vue de l'implantation possible d'un élevage, bien que des essais sur les espèces locales aient été mis en place. Cette espèce ne semble pas avoir colonisé les eaux libres (KEITH et al., 2002).

\section{DISCUSSION}

Le Tableau I donne le bilan des introductions d'espèces de poissons et de crustacés d'eau douce en Polynésie française. La définition d'une espèce acclimatée est tirée des travaux de SHAFLAND et LEWIS (1984), modifiée par KEITH et ALLARDI (1997), à savoir (1) espèce introduite : espèce dont la présence actuelle ou passée est liée à une action anthropique volontaire ou involontaire ; (2) espèce acclimatée : espèce introduite dont les populations se maintiennent naturellement.

Sur 11 espèces introduites en Polynésie française : 3 se sont acclimatées, 3 ne sont pour l'instant présentes que dans les élevages et 5 ne se sont pas acclimatées. 
Sur ces 11 espèces introduites, 6 l'ont été avant 1930 sous l'influence de la Société d'Etudes Océaniennes (S.E.O.) qui avait suivi avec attention ce qui avait été réalisé en métropole par la Société Nationale d'Acclimatation (sur celles-ci, on note que 2 espèces ont été plutôt introduites à des fins de lutte biologique, 2 à des fins de pêche et 2 par « curiosité scientifique ») ; enfin 5 l'ont été sous l'impulsion du développement de projets aquacoles.

\section{Tableau I}

Espèces introduites en Polynésie française.

\section{Table I}

Introduced species in French Polynesia.

\begin{tabular}{|c|c|c|c|}
\hline Espèces & $\begin{array}{l}\text { Date de } \\
\text { l'introduction }\end{array}$ & $\begin{array}{l}\text { Motivations de } \\
\text { l'introduction }\end{array}$ & $\begin{array}{l}\text { Archipel(s) où l'espèce } \\
\text { est présente }\end{array}$ \\
\hline \multicolumn{4}{|l|}{ ACCLIMATÉES } \\
\hline \multicolumn{4}{|l|}{ Poissons } \\
\hline Poecilia reticulata & 1920 & LB & Société, Australes \\
\hline Poecilia sphenops & 1970 & PA & Société \\
\hline \multicolumn{4}{|l|}{ Cichlidae } \\
\hline Oreochromis mossambicus & 1950 & PA & Société, Australes, Gambier \\
\hline \multicolumn{4}{|l|}{ NON ACCLIMATÉES } \\
\hline \multicolumn{4}{|l|}{ Poissons } \\
\hline Oncorhynchus mykiss & 1927 & PE & - \\
\hline \multicolumn{4}{|l|}{ Cyprinidae } \\
\hline Cyprinus carpio & 1925 & PE & - \\
\hline \multicolumn{4}{|l|}{ Poeciliidae } \\
\hline Gambusia affinis & 1925 & LB & - \\
\hline \multicolumn{4}{|l|}{ Ictaluridae } \\
\hline Ictalurus sp. & 1925 & CS & - \\
\hline \multicolumn{4}{|l|}{ Centrachidae } \\
\hline Micropterus salmoides & 1926 & CS & - \\
\hline \multicolumn{4}{|l|}{ EN ÉLEVAGES } \\
\hline \multicolumn{4}{|l|}{ Poissons } \\
\hline Chanos chanos & 1971 & PA & Tuamotu \\
\hline \multicolumn{4}{|l|}{ Centropomidae } \\
\hline Lates calcarifer & 1980 & PA & Société \\
\hline \multicolumn{4}{|l|}{ Crustacés } \\
\hline Macrobrachium rosenbergii & $>1970$ & PA & Société \\
\hline
\end{tabular}

LB : lutte biologique ; PE : pêche ; PA : projet aquacole ; CS : curiosité scientifique. 


\section{Quels sont les impacts des espèces introduites et acclimatées en Polynésie française?}

Les espèces introduites qui se sont acclimatées colonisent surtout le cours inférieur des rivières et les étangs littoraux saumâtres, elles ne dépassent jamais la première cascade sauf dans les cas particuliers comme le lac Vahiria où l'homme a pu les introduire directement en altitude (KEITH et al., 2002).

Les espèces les plus rhéophiles de Polynésie française, qui sont souvent endémiques et qui colonisent surtout les zones amonts (Lentipes, Sicyopus, Sicyopterus...) pourraient donc ne pas être systématiquement affectées par la présence des espèces introduites, cependant les espèces indigènes ont toutes une phase larvaire marine planctonique puis une phase de colonisation de la rivière à partir de l'embouchure, et la prédation excercée par les tilapias ainsi que l'occupation des niches par les taxons introduits pourraient avoir un impact défavorable sur le recrutement de ces espèces et donc sur les stocks.

Le guppy, introduit à des fins de lutte biologique pour limiter la prolifération des moustiques a été peu étudié en Polynésie mais il l'a été dans d'autres régions du monde. Ainsi, LEVER (1997) constate qu'il mange les œufs des autres poissons et est responsable de la régression d'espèces indigènes dans certains pays. Dans certains cours d'eau du sud-ouest des Etats-Unis, l'introduction du guppy a particulièrement affecté les populations de poissons endémiques (COURTENAY et STAUFFER, 1990). En particulier, les guppys implantés à Preston dans le Nevada ont entraîné la régression d'un petit cyprinodonte indigène Crenichthys baileyi (DEACON et al., 1964).

En Australie, dans le Queensland, là où les guppys sont nombreux, les espèces endémiques ont tendance à se raréfier (ARTHINGTON, 1991). Mc KAY (1986-1987) notait que, même placé dans des rivières où existait déjà la gambusie, non seulement le guppy survivait, mais il devenait aussi l'espèce dominante, préférant les eaux les plus courantes et laissant les eaux stagnantes marécageuses à la gambusie.

En Papouasie, en raison de sa rapide reproduction, il est considéré comme une peste. Dans la région de Port Moresby, il a fait disparaître tous les endémiques (ALLEN, 1991).

A Hawaii, FONT (1997) a mis en évidence la présence de 4 espèces d'helminthes parasites issus des Poeciliidae introduits et susceptibles d'avoir des conséquences sur les espèces indigènes. Cependant, FITZSIMONS et al. (1997) ont montré que lorsque le débit est supérieur à $20 \mathrm{~cm} / \mathrm{s}$, les Poeciliidae introduits disparaissent ou ne s'installent pas et, de fait, les helminthes parasites qu'ils transportent ne peuvent contaminer les espèces indigènes.

Le tilapia n'a pas non plus pour l'instant fait l'objet d'études spécifiques en Polynésie française, néanmoins, cette espèce et son impact ont été très étudiés à travers le monde.

En Inde, le tilapia du Mozambique est l'espèce de poisson la plus controversée. Aucune autre espèce n'a jamais eu autant de conséquences écologiques (SREENIVASAN, 1991). Elle fut introduite pour fournir des protéines à la population. RAVICHANDRA REDDY et al. (1990) signalent que bien que le tilapia ait permis un certain développement des pêcheries, son introduction dans certains lacs a entraîné la régression de plusieurs espèces locales de cyprinidés. D'autre part, le tilapia se reproduit tout au long de l'année à la différence des autres espèces dont la reproduction est saisonnière, ce qui lui donne un net avantage compétitif. Ceci a été constaté aussi dans d'autres lacs où les Puntius ont disparu ou diminué en taille et en nombre (CHANDRASEKHARAIAH, 1989 ; BANERJI et SATISH, 1989). 
Au Sri Lanka, l'introduction du tilapia du Mozambique, en fournissant une nouvelle et importante ressource alimentaire, a provoqué aussi, par l'augmentation de l'effort de pêche, une augmentation de la prise d'espèces indigènes qui n'étaient pas commercialisées auparavant (FERNANDO, 1971). Cependant, dans cette région il ne semble pas en compétition avec les espèces locales (PAYNE, 1987).

En Thaïlande, l'espèce est considérée comme une espèce nuisible et l'on pense qu'elle entre en compétition dans les étangs dévolus à l'aquaculture avec la crevette Penaeus merguiensis (PIYAKARNCHANA, 1989).

En Namibie, bien qu'il n'y ait pas eu jusqu'à présent d'hybridation constatée entre le tilapia du Mozambique et les espèces indigènes de tilapia, DE MOOR et BRUTON (1988) supposent qu'elle est possible avec $O$. macrochir et $O$. andersonii. SCHRADER (1985) pense que la compétition alimentaire et sur les frayères est réelle.

En Afrique du Sud, dans la rivière de Jan Diesels, le tilapia du Mozambique est en compétition avec des Barbus indigènes (CRASS, 1969).

Aux Etats-Unis, KNAGGS (1977) indique un déclin dans les populations de poissons indigènes dans la rivière de San Gabriel (Californie) qu'il attribue à l'introduction puis à l'augmentation des populations de tilapias.

Pour l'instant, les impacts du tilapia du Mozambique en Mélanésie, Polynésie et Micronésie, de nature écologique ou socio-économique, sont souvent cités comme nombreux mais sont difficiles à quantifier car leurs impacts sur la faune et la flore locale, l'aquaculture ou les pêcheries sont peu étudiés.

L'un des cas les mieux documentés concerne l'impact sur des oiseaux indigènes. Aux Tongas, à Niuafo'ou, l'eau du cratère colorée en vert par une algue s'est éclaircie avec l'introduction du tilapia du Mozambique. La population de canards indigènes s'est ensuite progressivement effondrée (SCOTT, 1993).

Dans le lac Susupe à Saipan dans les îles Mariannes, la disparition progressive de la gallinule endémique (Gallinus chloropus guami) est dûe en partie à sa compétition avec le tilapia (STINSON et al., 1991).

A Tongatapu, aux Tongas, le tilapia du Mozambique gêne l'élevage du mulet, Mugil cephalus. A Fanefuti (Tuvalu), il empêche celui du Chanos chanos (UWATE et al., 1984). De même à Nauru où l'élevage traditionnel du Chanos chanos a presque disparu en raison de l'introduction du tilapia (NELSON et ELDREDGE, 1991). A Tarawa et Kiribati il entre en compétition avec Chanos chanos pour la nourriture et les frayères (NELSON et ELDREDGE, 1991).

A Hawaii, en 1951, 60 petits tilapias du Mozambique en provenance de Singapour furent importés et introduits sur l'île d'Oahu. Quatorze survécurent et se reproduisirent avec succès dans les bassins du laboratoire de la "Fish \& Game Division». Les descendants furent envoyés sur d'autres îles et servirent à contrôler la végétation aquatique, à l'alimentation humaine ou à la pêche sportive (MACIOLEK, 1984). L'espèce entre en compétition sur ces îles avec le mulet cabot (Mugil cephalus) (ELDREDGE, 1994).

A l'atoll Fanning et l'île Washington, le tilapia du Mozambique occupe l'estuaire à marée basse, gagnant les eaux peu profondes lorsque la marée monte. Les poissons se font ainsi souvent piéger dans des flaques. C'est ce que nous avons pu aussi constater à 
Moorea en Polynésie française dans le lac de l'aéroport (KEITH et al., 2002). Pour les pêcheurs locaux, le tilapia a provoqué un important déclin d'espèces locales telles que Mugil cephalus, Mugil engeli, Chanos chanos et Albula vulpes qui utilisent les estuaires comme nurseries (LOBEL, 1980).

Dans certaines régions, après introduction puis acclimatation, le tilapia du Mozambique a parfois régressé jusqu'à quasi-disparition, sans que les causes n'en soient identifiées. Dans les îles Cook, par exemple, les populations ont d'abord explosé au niveau démographique, puis ont énormément régressé, rendant sa pêche aléatoire (NELSON et ELDREDGE, 1991).

\section{CONCLUSION}

De nombreuses introductions d'espèces de poissons et de crustacés d'eau douce ont eu lieu en Polynésie française, mais pour l'instant peu se sont acclimatées. L'impact des espèces acclimatées est mal connu, mais probablement non négligeable sur certaines rivières déjà fragilisées.

Au total, les rivières de la Polynésie française abritent 37 espèces de poissons et 18 de crustacés décapodes soit 55 espèces. Parmi celles-ci, 17 espèces sont endémiques soit $31 \%$ (40,5 \% pour les poissons et $11 \%$ pour les crustacés) et 3 sont introduites et acclimatées (soit $5 \%$ ).

La richesse spécifique est maximale dans l'archipel de la Société (20 espèces de poissons et 14 de crustacés décapodes) et dans l'archipel des Australes (20 espèces de poissons et 13 de crustacés). Ce sont ces deux archipels qui concentrent surtout les espèces introduites, celles-ci n'ayant pas encore été observées aux Marquises (KEITH et al., 2002).

Les Marquises, avec un taux d'endémisme chez les poissons d'eau douce voisin de $64 \%$ et chez les Crustacés décapodes de 14,5\%, constituent un archipel d'une grande valeur patrimoniale. Les Australes, et l'île de Rapa en particulier, ont aussi un taux d'endémisme important : $21 \%$ chez les poissons d'eau douce et 7,5\% chez les crustacés.

La faune d'eau douce polynésienne n'est donc pas encore perturbée dans toutes les îles. La répartition des espèces et la présence d'endémiques, telle que l'on peut actuellement l'observer dans les milieux indemnes d'introductions, correspond à l'intégration de toutes les étapes d'une évolution complexe (colonisation, isolement, spéciation...). La compréhension du fonctionnement des écosystèmes aquatiques est liée à leur préservation et à celle des cours d'eau polynésiens. Préserver les rivières polynésiennes de toute introduction, c'est se donner les premiers éléments pour préserver l'avenir des espèces et pour élaborer des mesures de gestion et de conservation adaptées.

\section{REMERCIEMENTS}

L'auteur tient à remercier E. Vigneux (CSP), G. Marquet et René Galzin (CRIOBE/EPHE). 


\section{RÉFÉRENCES}

ADAMSON A.M., 1935. Non marine invertebrate fauna of the Marquesas (exclusive of insects). Occasional Papers Bernice P. Bishop Museum., 11 (10), 1-39.

ALLEN G.R., 1991. Freshwater fishes of New Guinea. Madang, PNG: Christensen Research Institute.

ARTHINGTON A.H., 1991. Ecological and genetic impacts of introduced and translocated freshwater fishes in Australia. Canadian Journal of Fisheries and Aquatic Sciences, 48 (suppl.1), 33-43.

BANERJI S.R., SATISH M.S., 1989. Changes brought about by the introduction of exotic fishes in Bihar. In : JOSEPH M.M. (ed.), Exotic Aquatic Species in India. Asian Fisheries Society Special Publication, 1, 97-99.

BOUVIER E.L., 1925. Recherches sur la morphologie, les variations, la distribution géographique des crevettes de la famille des Atyidés. Enc. Entomol., Paris, 4, 1-370.

BROWN T.W. 1976. Preliminary report on the potential for Chanos chanos (milkfish) culture in French Polynesia. 13 september 1976.

CHANDRASEKHARAJAH H.N., 1989. Status of exotic fish culture in Karnataka. In : JOSEPH M.M. (ed.) Exotic Aquatic Species in India. Asian Fisheries Society Special Publication, 1, 75-77.

COURTENAY W.R. Jr., STAUFFER J.R. Jr, 1990. The introduced fish problem and the aquarium fish industry. Journal of the world Aquaculture Society, 21, 145-159.

CRASS R.S., 1969. The effects of land use on freswater fish in South Africa, with particular reference to Natal. Hydrobiologia, 34, 38-56.

CROSSLAND J., GRANDPERRIN R., 1979. Fisheries Directory of the South Pacific Commission Region. South Pacific Commission $\mathrm{n}^{\circ}$ 1. Nouméa, New Caledonia.

DE MOOR I.J., BRUTON M.N., 1988. Atlas of Alien and Translocated Indigenous Aquatic Animals in Southern Africa. South African National Scientific Programmes Report, $144,310 \mathrm{p}$.

DEACON J.E., HUBBS C., ZAHURANEC B.J., 1964. Some effects of introduced fishes on the native fish fauna of Southern Nevada. Copeia, 1964, 384-388.

DEVAMBEZ L.C., 1964. Le Tilapia dans le Pacifique Sud. Bulletin du Pacifique Sud, 14 (4), 13,14 et 53.

EDMONDSON C.H., 1935. Atyidae of southern Polynesia. Occasional Papers Bernice P. Bishop Museum., 11 (3), 1-19.

EDMONDSON C.H., 1951. Some central Pacific crustacean. Occasional Papers Bernice P. Bishop Museum., 20 (13), 183-243.

EGE V., 1939. A revision of the genus Anguilla Shaw: a systematic, phylogenetic and geographical study. The Carlsberg foundation's oceanographical expedition round the world 1928-30 and previous « Dana »-expeditions. Dana Report, 16 (3), 1-256.

ELDREDGE L.G., 1994. Perspectives in aquatic exotic species management in the Pacific Islands. Vol. I, I Introductions of commercially significant organismes to the Pacific Islands. Noumea, New Caledonia, South Pacifique Commission, $127 \mathrm{p}$.

FERNANDO C.H., 1971. The role of introduced fish on fish production in Ceylon's freshwaters. In : DUFFAY E.B., WATT A.S. (Eds), The Scientific Management of Animal and Plant Communities for Conservation, 295-310. Oxford, Blackwell.

FITZSIMONS J., SCHOENFUSS H.L., SCHOENFUSS T.C., 1997. Significance of unimpeded flows in limiting the transmission of parasites from exotics to hawaiian stream fishes. Micronesica, 30 (1),117-125.

FONT W.F., 1997. Improbable colonists: Helminth parasites of freshwater gobies on an oceanic island. Micronesica, 30 (1), 105-145.

FOURMANOIR P., GRIESSINGER J.M., PLESSIS Y., 1974. Faune ichtyologique des Gambier. Cahier du Pacifique, 18 (2), 543-559.

FOWLER H.W., 1925. Fishes of Gwam, Hawaï, Samoa and Tahiti. Bernice P. Bishop Museum Bulletin, 22, 1-38. 
FOWLER H.W., 1932. Freshwater fishes from the Marquesas and Society islands. Occasional Papers Bernice P. Bishop Museum., 9 (25), 1-11.

FUCHS J., 1987. Growth of introduced larvae and fingerlings of sea bass (Lates calcifer) in Tahiti. In : management of wild and cultured seabass/barramundi. ACIAR Monograph, 20, 189-192.

HOBBS H.H. Jr, HART C.W. Jr, 1982. The shrimps genus Atya (Decapoda: Atyidae). Smithsonian contribution to zoology, 364, 1-143.

HOLTHUIS L.B., 1950. The decapoda of the Siboga expedition. The Palaemonidae collected by the Siboga and Snellius expeditions with remarks on other species I. Subfamily Palaemonidae. Siboga Expeditie, Leiden, 39, 1-268.

HOLTHUIS L.B., 1952. On some Indo-Westpacific Palaemoninae (Crustacea decapoda Caridea). Zoologishe mededelingen, 31 (18), 201-211.

HOLTHUIS L.B., 1980. Shrimps and prawns of the world, an annnoted catalogue of species of interest to fisheries. FAO Fisheries Synopsis N¹25 (1). $230 \mathrm{p}$.

KEITH P., ALLARDI J., 1997. Bilan des introductions de poissons d'eau douce en France. Bull. Fr. Pêche Piscic., 344/345, 15-32.

KEITH P., VIGNEUX E., 1997. Inventaire des poissons et crustacés d'eau douce de Polynésie française. Rap. MNHN, EPHE, CSP, Del. Env. Polynésie, $110 \mathrm{p}$.

KEITH P., VIGNEUX E., 2002. Revue des crustacés Atyidae et Palaemonidae de Polynésie française avec description d'une nouvelle espèce de Macrobrachium. Bull. Fr. Pêche Piscic., 364, 121-145.

KEITH P., VIGNEUX E., FEUNTEUN E., 2000. Rapport de la mission d'inventaire des poissons et des crustacés d'eau douce effectuée aux îles Marquises. MNHN/EPHE, $11 \mathrm{p}$.

KEITH P., VIGNEUX E., MARQUET G., 2002. Atlas des poissons et des crustacés d'eau douce de Polynésie française. Patrimoines naturels, sous presse.

KENT G., 1980. The politics of Pacific Island fisheries. Boulder, Colorado, Westview Press.

KNAGGS E.H., 1977. Status of the genus Tilapia in California's estuarine and marine waters. California-Nevada Wildlife Transactions, 1977, 60-67.

LEVER C., 1997. Naturalized fishes of the world. Academic Press ed., London, 408 p.

LOBEL P.S., 1980. Invasion by the Mozambique tilapia (Sarotherodon mossambicus, Pisces; Cichlidae) of a Pacific atoll marine ecosystem. Micronesica, 16, 349-355.

MACIOLEK J.A., 1984. Exotic fishes in Hawaï and other islands of Oceania. In : COURTENAY W.R. Jr, STAUFFER J.R. Jr (Eds.), Distribution, Biology and Management of Exotic fishes, 131-161. Baltimore, J. Hopkins University Press.

MARQUET G., 1991. Freshwater crustaceans of French Polynesia: taxonomy, distribution and biomass (Decapoda). Crustaceana, 61 (2), 126-140.

MARQUET G., 1993. Étude biogéographique de la faune d'eau douce de Polynésie française. Biogeographica, 69 (4), 157-170.

MARQUET G., GALZIN R., 1992. Systématique, répartition et biomasse des poissons d'eau douce de Polynésie française. Cybium, 16 (3), 245-259.

MC KAY R., 1986-1987. It 's your problem too! Parts 3 and 4. The Australian introductions. Aquarium Life Australia, 2, 37-40.

NELSON S.G., ELREDGE L.G., 1991. Distribution and status of introduced cichlid fishes of the general Oreochromis and Tilapia in the islands of the South Pacific and Micronesia. Asian Fisheries Science, 4, 11-22.

PAYNE I.,1987. A lake perched on piscine peril. New Scientist, 115, 50-54.

PIYAKANCHANA T., 1989. Exotic aquatic species in Thailland. In : DE SIVA S.S. (ed.), Exotic Aquatic Organisms in Asia. Asian Fiheries Society Special Publication, 3, 119-124.

PLESSIS Y., 1980. Etude ichtyologique de Tubuaï, archipel des Australes (Polynésie). Cahier de l'Indo-Pacifique, 2 (3), 255-269.

PLESSIS Y., 1987. Etude ichtyologique de Rapa. DIRCEN SMCB, LEFRANCQ et Cie, Eds., Candé, 215-230.

PLESSIS Y., MAUGÉ L.A., 1978. Ichtyologie des îles Marquises. Cahier du Pacifique, 21, 215-235. 
POLL M., 1942. Les poissons de Tahiti recueillis par G.A. De Witte. Bulletin du muséum royal d'histoire naturelle de Belgique, 18 (61), 1-20.

PRESTON G.L., 1990. Brackish-water aquaculture in Tahiti. South Pacific Commission Fisheries Newsletter, 55, 36-40.

RANDALL J.E., 1973a. Tahitian fish names and preliminary checklist of the fishes of the Society Islands. Occasional Paper Bernice P. Bishop Museum., 24 (11), 167-203.

RANDALL J.E., 1973b. Rapan fish names and a preliminary checklist of the fishes of the Society Islands. Occasional Paper Bernice P. Bishop Museum., 24 (15), 291-306.

RANDALL J.E., SMITH C.L., FEINBERG M.N., 1990. Report on fish collections from Rapa, French Polynesia, American museum novitates, 2966, $44 \mathrm{p}$.

RAVICHANDRA REDDY S., NIJAGUNA, G.M., SHAKUNTALA K., 1990. The impact of the introduction of Oreochromis mossambicus (Peters) on the native carps. In : The Biology and Conservation of Rare Fish. Papers from an international symposium of The Fisheries Society of the British Isles.

ROUGIER E., 1926a. Essai de pisciculture. Bulletin de la Société des Etudes Océaniennes, 11,16-20.

ROUGIER E., 1926b. Pisciculture. Bulletin de la Société des Etudes Océaniennes, 12, 40-41.

ROUGIER E., 1926c. Moustiques et poissons anti-moustiques. Bulletin de la Société des Etudes Océaniennes, 13, 65-66.

ROUGIER E., 1928. Pisciculture. Bulletin de la Société des Etudes Océaniennes, 26, 107-109.

SCHRADER H.J., 1985. Invasive alien fishes of South West Africa/Namibia. South African National Scientific Programme Report, 199, 35-40.

SCOTT D.A., 1993. A Directory of Wetlands in Oceania. Slimbridge, England: IWRB; Kuala Lumpur, Malaysia: AWB.

SEURAT L.G., 1934. La faune et le peuplement de la Polynésie française. Mémoire de la Société de Biogéographie, 4, 41-74.

SHAFLAND P.L., LEWIS W.M., 1984. Terminology associated with introduced organisms. Fisheries, 9, 17-18.

SPC, 1975. Report. $8^{\text {th }}$ Regional Technical Meeting on Fisheries. Noumea, New Caledonia. 20-24 october 1975. SPC/Fisheries13-17 March 1978. SPC/Fisheries 10/Report.

SPC, 1978. Report. $10^{\text {th }}$ Regional Technical Meeting on Fisheries. Noumea, New Caledonia. 13-17 March 1978. SPC/Fisheries 10/Report.

SREENIVASAN, A. 1991. Transfers of freshwater fishes into India. In : Ecology of Biological Invasions in the Tropics, RAMAKRISHNAN P.S. (ed.). New Delhi: International Scientific Publications (International Journal of Ecology and Environmental Sciences), suppl 16, 131-138.

STINSON D.W., RITTER M.W., REICHEL J.D., 1991. The Mariana common morrhen: decline in an island endemic. Condor, 93, 38-43.

TOKORAGI, L. 1978. Milkfish culture in French Polynesia. Appropriate Technology, 4 (4), 23-25.

UWATE K.R.P., KUNATUBA B. RAOBATI, TENAKANAI C., 1984. A review of aquaculture activities in the Pacific Islands region. Pacific Islands Deveopment Program, EastWest Center, Honolulu.n.p.

VAN PEL H., DEVAMBEZ L.C., 1957. The fisheries industry of French Polynesia. South Pacific Commission, Noumea, New Caledonia.

VILLALUZ D.K., 1972. Aquaculture possibilities in some islands of the South Pacific. Report prepared for the South Pacific Islands Fisheries Development Programme. FAO DP/RAS/69/102/12. 